\title{
Interaction of waveguide and localized modes in a phononic crystal
}

\author{
S. Benchabane ${ }^{1}$, A. Khelif $^{1}$, A. ChoujaA ${ }^{1}$, B. Djafari-Rouhani ${ }^{2}$ and V. Laude ${ }^{1}$ \\ 1 Département LPMO, Institut FEMTO-ST, CNRS UMR 6174, Université de Franche- \\ Comté - 32 avenue de l'Observatoire, F-25044 Besançon cedex, France \\ 2 Laboratoire de Dynamique et Structure des Matériaux Moléculaires, CNRS UMR 8024 \\ - Université de Lille I, F-59655 Villeneuve d'ascq cedex, France
}

PACS. 43.20.+g - General linear acoustics.

PACS. 43.35.+d - Ultrasonics, quantum acoustics, and physical effects of sound.

PACS. 63.20.-e - Phonons in crystal lattices.

\begin{abstract}
The transmission properties of phononic crystal waveguides with grafted stub resonators were measured. The phononic crystal consists of a two-dimensional periodical array of steel rods immersed in water. Waveguides for acoustic waves are created by removing a line defect and stubs are formed by removing rods from the sidewalls of the waveguide. Depending on the stub geometry, definite wavelengths are reflected from the stub creating a one-dimensional bandgap within the waveguide transmission spectrum, the bandwidth of which can be controlled by arranging a proper sequence of stubs. These effects are interpreted as the interaction of propagating waveguide modes with localized stub resonances.
\end{abstract}

A great deal of research effort is currently being devoted to the study of acoustic or elastic waves propagation in periodically structured materials, such as the so-called phononic crystals [1-5]. Phononic crystals are somehow the elastic analogous to photonic crystals. They are made of two- or three dimensional periodical repetitions of different solids or fluids which exhibit absolute stop bands in the transmission spectrum of acoustic waves. The location and width of these band gaps result from a large contrast in the value of elastic constants and/or mass density of the constitutive materials. These structures hence have potential applications as acoustic filters or waveguides, among others. Such materials would be very useful for applications requiring the spatial localization of acoustic waves. Efficient guiding and bending of acoustic and elastic waves have both been studied theoretically $[6,7]$ and demonstrated experimentally [8-11]. We are here dealing with the possibility of altering the transmission characteristics of an acoustic waveguide managed through a phononic crystal by grafting stub tuners on it. A typical waveguide is formed by removing a line of rods and possesses confined propagation modes within a large frequency range. By grafting an additional defect, or stub, to the sidewall of the waveguide, it was predicted theoretically that the transmission spectrum can be dramatically altered $[12,13]$. Indeed, the interaction between the stub and the waveguide can generate spectrally sharp zeros in the transmission spectrum of the waveguide occurring at the resonance modes of the stub. The zeros occur

(c) EDP Sciences 

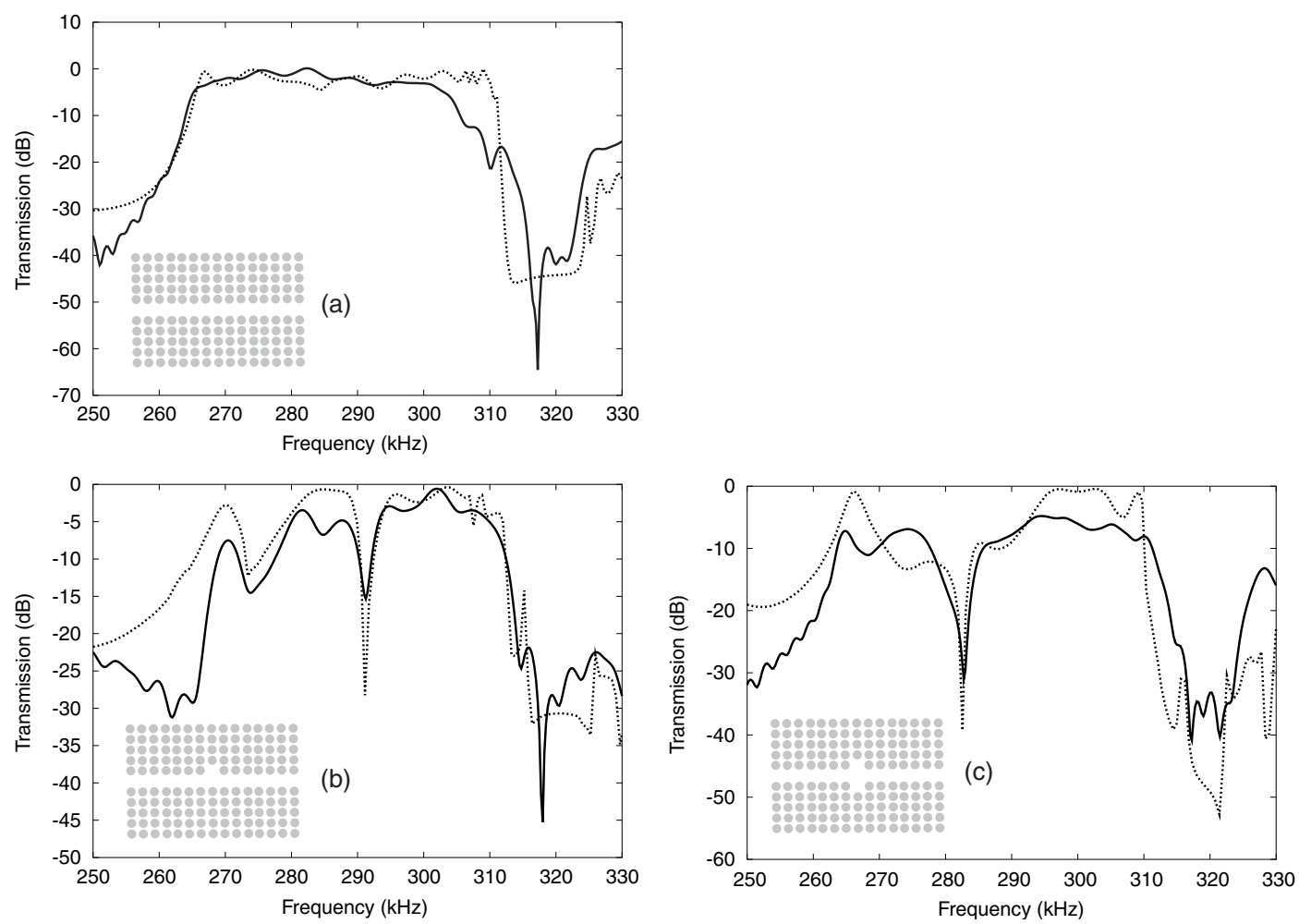

Fig. 1 - Experimental (solid line) and calculated (dashed line) transmission power spectra through (a) the plain waveguide, and the waveguide with a grafted (b) single or (c) symmetrical stub (side branch). The insets show the geometry of the samples.

at the resonance modes of the stubs and a sequential arrangement of several stubs broadens a zero of transmission into a band gap with a controllable bandwidth. Thus, we can create a band gap for a one-dimensional system constituted by the waveguide within the phononic crystal. The propagation in this system behaves qualitatively in the same manner as explained by simple analytical models where longitudinal waves propagate in slender tubes consisting of equidistant side branches [12]. Also, the idea of a strong resonance between a periodic series of stubs and the wave propagating along a guide is related to the wave interaction between the periodic roughness of the guide walls and the guided wave [6]. In this letter, we investigate experimentally the occurrence of these effects for a waveguide created in a two-dimensional phononic band gap material. The phononic crystal consists of a two-dimensional periodical array of $2.5 \mathrm{~mm}$-diameter steel rods, which act as strong acoustic scatterers, immersed in water. The rods are long enough with respect to a period $(150 \mathrm{~mm})$ to be considered infinite if the incident wave is propagating perpendicularly to the rods. The square lattice constant is $3 \mathrm{~mm}$, leading to a filling fraction of $55 \%$. Such an arrangement has previously been shown to present a full band gap in the $250 \mathrm{kHz}-325 \mathrm{kHz}$ frequency range [10]. The experimental setup itself is based on the ultrasonic immersion technique as described in detail in Ref. [10].

A straight waveguide is first formed by removing one row of rods. The measured transmis- 




Fig. 2 - Calculated longitudinal displacement field averaged over one period at frequencies 291 and $283 \mathrm{kHz}$ for (a) a one-period long and (b) a symmetrical stub, respectively. Grey levels are representative of the amplitude and range from black for negative values to white for positive values.

sion through this plain waveguide is shown in fig. 1a. The structure used here is 20-period, that is $60 \mathrm{~mm}$ long. The guiding band starts around $260 \mathrm{kHz}$ and ends at about $317 \mathrm{kHz}$, covering more than $75 \%$ of the perfect crystal bandgap. The experimental results are in good agreement with the theoretical predictions made using the finite difference time domain (FDTD) method, described in previous papers devoted to phononic crystals $[6,7,14,15]$. For such structures, we previously observed that the propagation is unimodal over most of the guiding band, and that the acoustic wave is strongly confined within the waveguide and is guided with weak losses [11]. This strong spatial confinement is the basis for the achievement of an efficient interaction between the waveguide and grafted resonators (branch stub).

Stubs are then defined along the guide by removing one or several rods. The stub modes are evanescent and strongly localized by the surrounding phononic crystal. Acoustic energy entering the stub exits after some delay time either to the left or to the right of the waveguide, and is transported away by the unique waveguide mode. When a one-period long branch is grafted on the side of the waveguide, fig. 1b, two dips appear in the transmission spectrum at frequencies around 274 and $291 \mathrm{kHz}$ respectively, the second dip being sharper than the first one. This difference in depth can be explained by the relatively close position of the first dip with respect to the beginning of the guiding band itself, where the transmission of the straight waveguide has not reached its maximum value yet. Additional experiments with different resonator lengths (namely two and three periods in the direction orthogonal to the waveguide) have confirmed that changing the length of the resonator modifies the resonant frequencies, that is, those which will not be transmitted. This behavior is still noticeable in the case of a symmetrical single stub as depicted in fig. 1c. The transmission spectrum in fig. 1c shows a main resonant frequency around $283 \mathrm{kHz}$.

The presence of a stub has a significant influence on the propagation of acoustic waves through the waveguide. It should be pointed out that the interaction of acoustic waves with stubs have been studied in the frame of a simple analytical model [12] in which longitudinal waves propagate in slender tubes consisting of equidistant side branches. This calculation predicts the existence of zeros of transmission whose frequencies are given in relation with the resonant frequencies of the side branches that depend upon their length. Such a simple model can even give a good account of the calculated transmission in a phononic crystal constituted by air cylinders in water [7]. In our present study, the transmission spectra through stubbed waveguides also display several zeros of transmission; nevertheless, a full numerical calculation is now required to obtain a good agreement with experiments.

The observed transmission spectra can qualitatively be explained by the fact that for frequencies close to stub resonance frequencies, stub modes are excited and can transfer the 


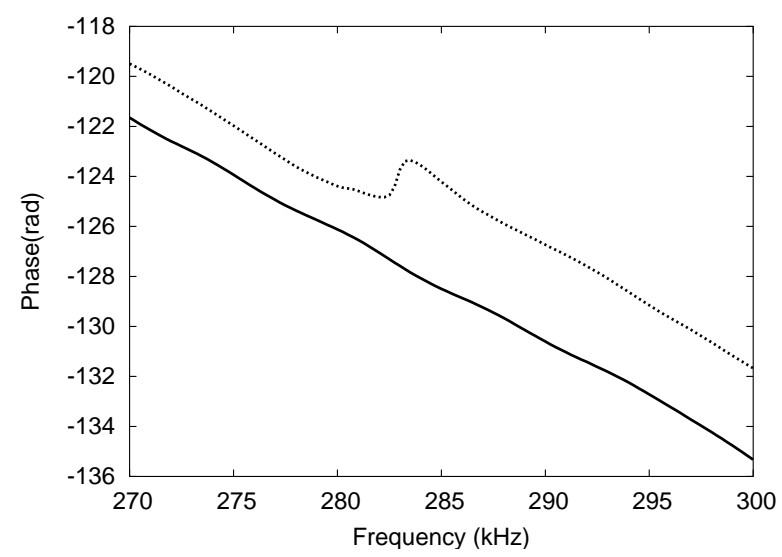

Fig. 3 - Experimental phase versus frequency measurements for the bare waveguide (solid line) and for the guide with a grafted symmetrical stub (dashed line).

energy back to the waveguide with some delay. For certain frequencies, this cancels the transmission of the incident waveguide mode. The wave is then reflected and returned back to the entrance of the guide. At other frequencies, the influence of the stub on the incident wave is negligible; although the wave actually enters the resonator, it seems to travel unperturbed straight through the guide. A numerical illustration of such a behavior was performed by using the FDTD computation to simulate a monochromatic source of frequency 291 and $283 \mathrm{kHz}$. The calculated displacement fields for both the single and the symmetrical stubs are displayed in figs. $2 \mathrm{a}$ and $2 \mathrm{~b}$ respectively, and clearly demonstrate that at these frequencies the stub acts as a localized mirror.

The phase of the acoustic wave is also an interesting parameter to take into account as it provides a direct measurement of the dispersion relation of the medium, and is plotted in fig. 3 . The waveguide is unimodal and the wavevector $k$ can be considered a linear function of the frequency within the guiding band. The phase shift hence gives a direct measurement of the dispersion relation $k(\omega)$ of the guided mode. The estimated group velocity of the guided mode is $830 \mathrm{~ms}^{-1}$, approximately, which is significantly smaller than the longitudinal velocity in water, $1490 \mathrm{~ms}^{-1}$. Phase measurements for both the plain waveguide and the waveguide with a grafted symmetrical side branch exhibit the same slope, but the introduction of the stub causes a phase jump to occur at its resonant frequency. This is linked to the reflection/transmission coefficient of the stub itself and reflects that this latter can be considered as a localized mirror. As the observed drops in transmission are not genuine zeros, a phase shift around $\pi / 2$ has been measured, though a $\pi$-value would be expected for a true transmission zero. Indeed, in a model calculation in which the transmission coefficient exactly goes through zero, the change of sign of this coefficient implies a phase jump of $\pi$. Such a result has been obtained in Ref. [12] as well as in other calculations dealing with photonic band gap materials $[16,17]$ and even with other types of excitations [16]. However, in calculations in which absorption phenomena are taken into account [18], as well as in microwave experiments in coaxial cables [18] and in photonic crystals [17] that exhibit dips instead of exact zeros of transmission, the antiresonance manifests itself by a fast but continuous decrease of the phase (instead of a jump) at the frequency of the dip in the transmission amplitude. The magnitude of the phase variation 

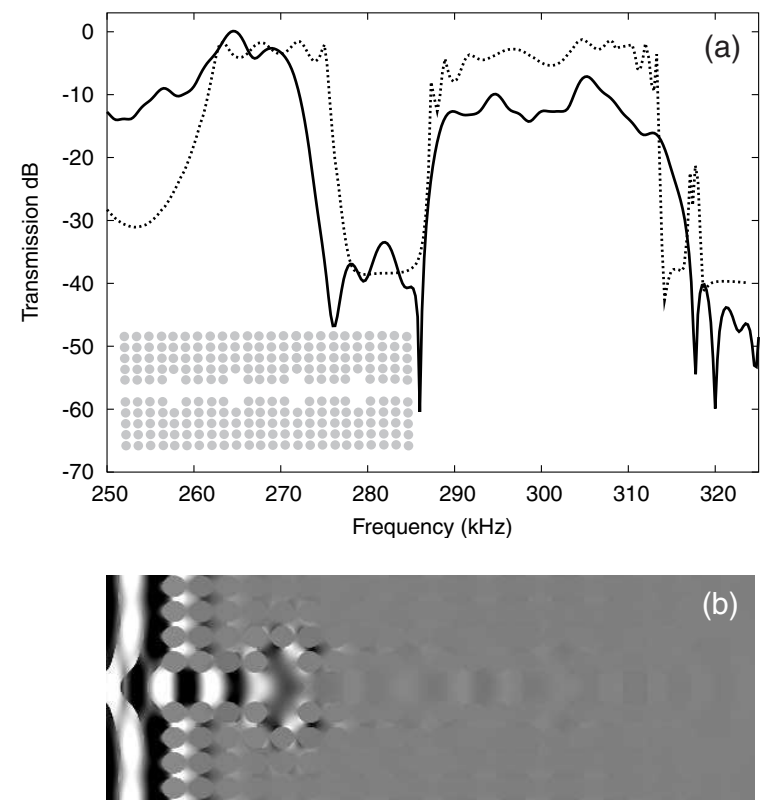

Fig. 4 - (a) Experimental (solid line) and calculated (dashed line) transmission power spectra along a sequence of symmetrical waveguide stubs. (b) Calculated longitudinal displacement field averaged over one period at a frequency of $285 \mathrm{kHz}$ for a sequence of symmetrical stubs.

remains in general significantly smaller than $\pi$, which is consistent with our observation.

The effects described above using a single stub can be enhanced by grafting a sequence of identical stubs along the waveguide. A sequence of 4 symmetrical stubs was added to the side of the waveguide and the length of the phononic crystal was increased to 22 periods $(66 \mathrm{~mm})$. In the example shown in fig. 4a, stubs are separated by 4 periods. The spacing between stubs was maintained larger than 3 periods in order to avoid evanescent coupling phenomena between the resonators. In the spectrum displayed in fig. 4a, a full, deeper bandgap opens for guided waves, forbidding the transmission of frequencies between 276 and $286 \mathrm{kHz}$. Fig. 4b shows the FDTD computation of the reflection of the guided wave on the sequence of stubs at a monochromatic frequency of $285 \mathrm{kHz}$. The increase in the attenuation of the forbidden frequencies observed here clearly results from the successive reflections of the wave on the stubs. It is then dependent on the overall number of stubs, that is, the larger this number the deeper the gap. It was also checked whether the gap for guided waves could be attributed, at least partly, to multiple reflections occurring between the stubs. To that purpose, a number of samples with varying separation between stubs, though larger than 3 periods, either arranged periodically or not, were prepared and measured. It was found that arranging or not the stubs in a periodical way and changing their separation did not have a noticeable influence on the transmission function, hence confirming that the number of stubs is the most important parameter to consider. However, it was verified that the transmission of a sequence of 4 stubs is not simply given by the fourth power of the transmission of a single stub, hence a sequence of stubs can not be simply viewed as a sequence of isolated spectral filters with the same resonance frequency. Instead, our interpretation is that the stub resonators are coupled along 
the waveguide, the only direction along which acoustic propagation occurs, and that this effect dominates over evanescent couplings that may arise through the surrounding phononic crystal. Such an arrangement is of great interest insofar as it allows controlling the position of a stop band by changing the design of the structure (that is, the stub length or its width), starting from a single basic structure. In the same way, it can help defining a particular guiding band, from the end of the secondary gap to the end of the guiding band (between 288 and $317 \mathrm{kHz}$ in our case).

As a conclusion, we have investigated the propagation of acoustic waves in a two-dimensional phononic crystal constituted by a square array of steel cylinders immersed in water, and containing waveguide stub resonators. It has been demonstrated that the presence of a single stub induces the apparition of narrow zeros of transmission in the waveguide spectrum. At these frequencies, the acoustic wave is efficiently reflected backwards from the stubs. The operating frequency of such structures can be tuned by changing the resonance mode of the stub, in particular by changing the length of the resonators. A sequence of side branches leads to the opening of a deep and tunable band gap. Potential device applications can be found in the fields of filtering or wavelength demultiplexing for instance.

The authors would like to express their gratitude to A. Reinhardt for fruitful discussions. One of the author (BDR) would like to thank Dr. E.H. El Boudouti for helpful discussions

\section{REFERENCES}

[1] Sigalas M. M. and Economou E. N., Solid State Commun., 86 (1993) 141.

[2] Kushwaha M. S., Halevi P., Dobrzynski L. and B. Djafari-Rouhani, Phys. Rev. Lett., 71 (1993) 2022.

[3] Montero de Espinosa F. R., Jimenez E. and Torres M., Phys. Rev. Lett., 80 (1998) 1208.

[4] Sanchez-Perez V., Caballero D., Martinez-Sala R., Rubio C., Sanchez-Dehesa J., Meseguer F., Llinares J. and Galves F., Phys. Rev. Lett., 80 (1998) 5325.

[5] Sigalas M. M. and Flory C. A., Phys. Rev. B, 65 (2002) 125209.

[6] Kafesaki M., Sigalas M. M. and Garcia N., Phys. Rev. Lett., 85 (2000) 4044.

[7] Khelif A., Djafari-Rouhani B., Vasseur J. O., Deymier P. A., Lambin P. and DobrzynSki L., Phys. Rev. B, 65 (2002) 174308.

[8] Torres M., Montero de Espinosa F., García-Pablos D. and García N., Phys. Rev. Lett., 82 (1999) 3054.

[9] T. Miyashita, Jpn. J. App. Phys., 41 (2002) 3170.

[10] Khelif A., Choujaa, A., Djafari-Rouhani B., Wilm M., Ballandras S. and Laude V., Phys. Rev. B, 68 (2003) 214301.

[11] Khelif A., Choujaa A., Benchabane S., Duafari-Rouhani B. and Laude V., Appl. Phys. Lett, 84 (2004) 4400.

[12] Kushwaha M. S., Akjouj A., Djafari-Rouhani B., Dobrzynski L. and Vasseur J. O., Solid State Commun., 106 (1998) 141.

[13] Khelif A., Djafari-Rouhani B., Vasseur J. O. and Deymier P. A., Phys. Rev. B, 68 (2003) 024302.

[14] Sigalas M. M. and Garcia N., J. App. Phys., 87 (2000) 3122.

[15] Vasseur J. O., Deymier P. A., Chenni B., Djafari-Rouhani B., Dobrzynski L. and Prevost D., Phys. Rev. Lett., 86 (2001) 3012.

[16] Vasseur J.O., Akjouj A., Dobrzynski L., Djafari Rouhani B. and El Boudouti, E.H., Surf. Sci. Rep., 54 (2004) 1. 
[17] Kondo T., Hangyo M., Yamaguchi S., Yano S., Segawa Y. and Ohtaka K., Phys. Rev. $B, 66$ (2002) 033111.

[18] El Boudouti E. H., Fettouhi N., Akjouj A., Djafari Rouhani B., Mir A., Vasseur J.O., Dobrzynski L. and Zemmouri J., J. Appl. Phys., 95 (2004) 1102. 